\title{
Long-term exposure to close-proximity air pollution and asthma and allergies in urban children
}

\author{
C. Pénard-Morand*\#, , C. Raherison ${ }^{+, \S}$, D. Charpin ${ }^{\dagger}$, C. Kopferschmitt**, \\ F. Lavaud ${ }^{\# \#}$, D. Caillaud " ${ }^{\text {"थ }}$ and I. Annesi-Maesano*,\#
}

ABSTRACT: The aim of this study was to evaluate the impact of urban air pollution, assessed through reliable indicators of exposure, on asthma and allergies in schoolchildren.

A validated dispersion model combining data on traffic conditions, topography, meteorology and background pollution was used to relate 3-yrs averaged concentrations of major urban pollutants at the sites of schools to skin prick tests, exercise-induced asthma and reported asthma and allergies in 6,683 children (9-11 yrs) attending 108 schools randomly selected in six French communities.

For the 4,907 children who had resided at their current address for the past 3 yrs, asthma (exercise induced, past year and lifetime) was significantly positively associated with benzene, $\mathrm{SO}_{2}$, particles with a $50 \%$ cut-off aerodynamic diameter of $10 \mu \mathrm{m}(\mathrm{PM} 10)$, nitrogen oxides $\left(\mathrm{NO}_{\mathrm{x}}\right)$ and $\mathrm{CO}$. In the same children, eczema (lifetime and past year) was significantly positively associated with benzene, $\mathrm{PM}_{10}, \mathrm{NO}_{2}, \mathrm{NO}_{\mathrm{x}}$ and $\mathrm{CO}$, lifetime allergic rhinitis with $\mathrm{PM}_{10}$ and sensitisation to pollens with benzene and PM10. Among the 2,213 children residing at their current address since birth, the associations persisted for lifetime asthma with benzene (adjusted OR per interquartile range $(95 \% \mathrm{Cl}) 1.3(1.0-1.9))$ and PM10 (1.4 (1.0-2.0)), and for sensitisation to pollens with volatile organic compounds (1.3 (1.0-1.9)) and PM10 (1.2 (1.0-1.9)).

Accurately modelled urban air pollution was associated with some measures of childhood asthma and allergies.

KEYWORDS: Air pollution, allergic rhinitis, allergic sensitisation, asthma, eczema, exposure assessment

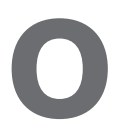
ver the past $35 \mathrm{yrs}$, the prevalence of asthma and allergies has increased considerably worldwide, particularly in industrialised countries [1]. This phenomenon is attributable to multiple factors. Air pollution has also considerably changed over the last decades. Since 1980, concentrations of $\mathrm{SO}_{2}$ and coarse particles, mainly emitted from industry, have significantly decreased in industrialised countries, while concentrations of traffic-related air pollutants (TAP) (nitrogen oxides $\left(\mathrm{NO}_{\mathrm{x}}\right)$, small particles and organic compounds) have increased steadily due to the growing number of motor vehicles, especially in urban areas where road traffic has become the main source of air pollutant emissions [2]. The evolution of the nature of the air pollution mix may have contributed to the epidemic of asthma and allergies [3].
Numerous epidemiological studies have demonstrated that short-term exposure to elevated concentrations of urban air pollutants can exacerbate pre-existing asthma [3, 4]. Nevertheless, adverse effects of long-term exposure are less clear. Whereas most of the initial studies described no relationship between the long-term exposure indicators and asthma and allergies [5-7], recent studies suggest that TAP may increase the prevalence of asthma and allergies [8-15], even if the results remained mixed [16].

The inconsistency in these results may be due to the use of different urban air pollution indicators, which are prone to exposure misclassification [17]. Some studies have used direct measurements outside the home, but most of the time only during periods of several weeks [9, 15]. Indeed, direct measurements of pollutants are
AFFILIATIONS

*INSERM, UMR-S 707

Epidemiology of Allergic and Respiratory Diseases,

\#Université Pierre et Marie Curie, Paris 6, UMR-S 707, Epidemiology of Allergic and Respiratory Diseases, "AgroParisTech, Institut des Sciences et Industries du Vivant et de I'Environnement, ENGREF, Paris, +Hôpital du Haut-Lévèque, Centre François Magendie, Pessac,

${ }^{\S}$ EA 3672, Public Health Institute, University of Bordeaux 2, Bordeaux, ${ }^{f}$ Hôpital Nord, Service de Pneumologie, Marseille, ${ }^{* *}$ Nouvel Hôpital Civil, Service de Pneumologie, Strasbourg,

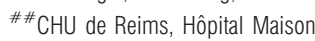
Blanche, Service de Pneumologie, Reims, and

"CHU de Clermont-Ferrand, Hôpital Gabriel Montpied, Service de Pneumologie, Clermont-Ferrand, France.

CORRESPONDENCE

C. Pénard-Morand

Epidemiology of Allergic and Respiratory Diseases, UMR S 707, INSERM-UPMC-Paris 6,

Medical School St. Antoine, 27 rue de Chaligny, 75571, Paris, Cedex 12 France

E-mail: celine.morand@

polytechnique.org

Received:

July 212009

Accepted after revision:

Dec 012009

First published online: Jan 142010 
more complex to implement when the number of subjects is high and the duration of exposure is long. Thus, most studies have used pollutant concentrations measured at central monitoring stations $[5,6]$. These measurements provide a good estimate of background concentrations; however, they underestimate the actual levels of TAP and fail to account for their local spatial variability within a community. To overcome these problems, numerous studies have included surrogate measures such as traffic density or distance to a major road $[7,13,14,16]$. A disadvantage of these exposure indicators is that they describe the pollutant emissions without taking dispersion conditions into account. A potential solution to obtain reliable estimates of urban air pollution exposure is the use of regression [18, 19] or dispersion models [20, 21]. However, to date, these models have rarely been implemented in population-based studies [8-10, 13, 22].

The objective of our cross-sectional study was to assess the role of urban air pollution in the prevalence of respiratory and allergic outcomes in a representative population-based sample of French schoolchildren living in six communities. There were three unique aspects of this study. 1) The use of long-term exposure estimates derived from one of the most sophisticated dispersion models capable of capturing the important smallarea variations in major traffic pollutants within communities. 2) The repeated sensitivity analysis among children residing at their current address since birth to reduce exposure misclassification. 3) The important number of potential confounders or modifiers that were taken into account. To date, this study has already reported associations between allergic health and air pollution indicators, namely long-term background concentrations from fixed stations [23], and 1-week measurements at school [24], which were used to assess long-term exposure to background air pollution and short-term exposure to proximity urban air pollution, respectively. These indicators are easy to obtain but are less reliable at assessing exposure to urban air pollution than the more accurate indicators built through a dispersion model that we propose [21]. The use of more reliable exposure indicators will reduce exposure misclassifications, which may have led to an attenuation of air pollution effect estimates.

\section{MATERIAL AND METHODS}

\section{Participants}

Between March 1999 and October 2000, 9,615 children (mean age $10.4 \mathrm{yrs}$ ) were recruited to participate in the French Six Cities Study, which was intended to estimate the prevalence and the severity of asthma and allergies, and to identify the associated risk factors. The sample was taken from all pupils in the 401 relevant classes from 108 schools randomly selected in the six French communities (Bordeaux, Clermont-Ferrand, Créteil, Marseille, Strasbourg and Reims), which were chosen for the contrast in their air quality.

\section{Protocol}

The study design included a standardised questionnaire, completed by the parents, and a clinical examination, conducted by a trained physician, according to an enhanced version of the International Study of Asthma and Allergies in Childhood (ISAAC) standardised protocol (ISAAC II) [25].
The questionnaire contained the core ISAAC questions on respiratory and allergic diseases [26], how these were managed and their potential risk factors. The clinical examination included a skin prick test (SPT) to common aeroallergens to assess allergic sensitisation and the standardised protocol of the run test to assess exercise-induced asthma (EIA) (refer to supplementary material). The research protocol was approved by the National Ethics Board (Comité Consultatif de Protection des Personnes se prêtant à des Recherches Biomédicales) and all children and parents gave written informed consent.

\section{Health outcomes}

Health outcomes were classified as follows. 1) Objective diagnosis during the clinical examination: EIA, defined as a decrease in peak expiratory flow after the run test $>10 \%$, and sensitisation to pollens, defined as at least one positive SPT to the tested pollens. 2) Reported symptoms during the last 12 months: asthma, rhinoconjunctivitis and eczema in the last 1 yr. 3) Reported diagnosis during life: lifetime asthma, allergic rhinitis and eczema. The methods used to assess the six reported outcomes through the replies to the ISAAC questions [23] are briefly summarised in the supplementary material.

\section{Exposure assessment}

Long-term exposure of the 6,683 children for whom complete health data were available (refer to the supplementary material for details on the participation rate) was assessed through 3-yr averaged (from 1998 to 2000) concentrations of major urban air pollutants (benzene, volatile organic compounds (VOC), $\mathrm{SO}_{2}$, particles with a 50\% cut-off aerodynamic diameter of $10 \mu \mathrm{m}$ (PM10), $\mathrm{NO}_{2}, \mathrm{NO}_{x}$ and $\mathrm{CO}$ ), estimated through the validated STREET 5 software (Targeting, Versailles, France), at the 108 school addresses. Home addresses were not available.

The implemented methods, as described previously [21], are briefly summarised below. To obtain annual mean concentrations of air pollutants, STREET, which is based on a dispersion model, combines data on both a regional component (background air pollution) and a local component, which depends on both traffic emissions and local dispersion conditions (topography and meteorology) in the modelled street segment (refer to supplementary material).

\section{Statistical analysis}

The analyses were restricted to the children residing at their current address for $\geqslant 3$ yrs $(n=4,907)$, in order to limit exposure misclassification. Sensitivity analyses were repeated among the children residing at their current address for $\geqslant 8$ yrs $(n=2,834)$, and among those residing at their current address since birth $(n=2,213)$.

Marginal models were applied to assess the associations between exposure to each air pollutant and health outcomes, in order to take into account the potential non-independence of data for children living in the same community. The parameters of the marginal models were estimated by the generalised estimating equation approach using SAS PROC GENMOD (SAS Institute, Cary, NC, USA) with an exchangeable working correlation structure using the community as a stratum. The models were adjusted for age, sex, older siblings, family history of allergy, parental education, mother's ethnic origin and potential sources of indoor pollution at home 
(smoking, mould or dampness, natural gas used for heating, cooking or water-heater, and pets). Details on the selection of adjustment factors including the study of the interactions between potential modifiers and air pollutants are available in the supplementary material. All odds ratios (ORs) are presented against interquartile range increases in air pollution concentrations.

Statistical significance was assessed using a two-sided test at a $5 \%$ level of significance. Regarding the power of the study, even after excluding children who changed residence during the last 3 yrs, our study was able to detect with a power of $80 \%$ (associated to a two-sided test and a type I risk $\alpha=5 \%$ ) an OR of 1.17 (and ORs of 1.19, 1.23, 1.25 and 1.36, respectively) for an outcome with a prevalence of $25 \%$ (and $20 \%, 12 \%, 10 \%$ and $5 \%$, respectively) among children with a low exposure to PM10 (low exposure: $n=2,447$; high exposure: $n=2,460$; the two categories of exposure low versus high were defined with respect to the median value of the distribution of the concentrations at schools). Version 9.1 of SAS System for Windows (SAS institute, Cary, NC, USA) was used for the analyses.

\section{RESULTS}

\section{Exposure to air pollutants}

Figure 1 shows the distribution of the pollutant concentrations calculated by STREET at the 108 schools. $64 \%$ of the schools had concentrations of $\mathrm{NO}_{2}$ above the World Health Organization guideline value of $40 \mu \mathrm{g} \cdot \mathrm{m}^{-3}$ and $76 \%$ of the schools had concentrations of PM10 above the World Health Organization guideline value of $20 \mu \mathrm{g} \cdot \mathrm{m}^{-3}$. Modelled concentrations of pollutants were highly correlated (refer to supplementary material). For each pollutant, concentrations differed considerably between the six communities (table 1). Concentrations are also shown separately for each school in the supplementary material. Associations between pollution exposure and potential confounders are described in the supplementary material.

\section{Population characteristics}

Table 2 summarises the characteristics of the study population, namely the 4,907 children residing at their current address for $\geqslant 3$ yrs. The 1,776 children excluded from the analysis did not differ significantly from the others in terms of the recorded characteristics; however, they did differ in the following four factors: 1) family history of allergy was less prevalent among them than among the study population $(33.5 \%$ versus $37.1 \%$, $\mathrm{p}=0.007)$; 2) they were less likely to have an older sibling $(41.5 \%$ versus $43.1 \%, p<0.001)$; 3 ) their parents were less frequently natives of metropolitan France $(\mathrm{p}<0.001)$; and 4$)$ there were fewer cases of mould or dampness in their home $(15 \%$ versus $17.6 \%, \mathrm{p}=0.01)$.

Table 3 shows the prevalence of health outcomes. When comparing the study population with the children excluded from the analysis no significant differences were found for all health outcomes listed in table 3 (refer to supplementary material).

The associations between health outcomes and potential confounders are described in the tables within the supplementary material. To summarise, health outcomes, except EIA, were related to sex and family history of allergy. Parental education, mother's ethnic origin and the four potential sources of indoor pollution at home were related to several health outcomes. No significant relationship was observed with older siblings.

\section{Associations between pollutants exposure and health outcomes}

The associations between pollutants exposure and health outcomes are described in table 3. An increment for an interquartile range of the concentrations of benzene, $\mathrm{SO}_{2}$, $\mathrm{PM} 10, \mathrm{NO}_{\mathrm{x}}$ and $\mathrm{CO}$ was associated with an increased risk for suffering from EIA. Interquartile range increases in concentrations of benzene, $\mathrm{SO}_{2}, \mathrm{NO}_{\mathrm{x}}$ and $\mathrm{CO}$ were associated with asthma in the past year; benzene, $\mathrm{SO}_{2}, \mathrm{PM} 10$ and $\mathrm{CO}$ with lifetime asthma; benzene, $\mathrm{PM} 10, \mathrm{NO}_{\mathrm{x}}$ and $\mathrm{CO}$ with past year eczema; $\mathrm{PM} 10, \mathrm{NO}_{2}, \mathrm{NO}_{x}$ and $\mathrm{CO}$ with lifetime eczema; benzene and PM10 with sensitisation to pollens; and PM10 with lifetime allergic rhinitis.

Sensitivity analyses repeated in the 2,834 children residing at their current address for $\geqslant 8$ yrs resulted in similar or even higher ORs. However, the confidence intervals were slightly wider, most likely due to the smaller sample size. The associations which remained significant or for which ORs were not stable are shown in figure 2. The associations of lifetime asthma with benzene, $\mathrm{SO}_{2}$ and $\mathrm{PM} 10$ and of sensitisation to pollens with PM10 were particularly robust. Restricting the analyses to the 2,213 children who have resided in their current home since birth yielded ORs similar to those obtained for the 2,834 8-yr resident children. In spite of the reduction of the sample size, the associations of lifetime asthma with benzene (1.3 (1.0-1.9); $\mathrm{p}=0.04)$ and PM10 (1.4 (1.0-2.0); $\mathrm{p}=0.05)$ remained significant. Moreover, a borderline significance existed in the case of the associations of sensitisation to pollens with VOC (1.3 (1.0-1.9); $p=0.07)$ and PM10 (1.2 (1.0-1.9); $\mathrm{p}=0.10)$.

Three additional analyses, including stratification on potential modifiers, application of a two-stage mixed model and application of a single-stage logistic model confirmed the results (refer to supplementary material).

\section{DISCUSSION}

For children residing at their current address for $\geqslant 3$ yrs, the risk for suffering from asthma, eczema, allergic rhinitis and sensitisation to pollens was significantly higher in neighbourhoods of schools with higher concentrations of some of the major urban pollutants, mainly influenced by traffic emissions. The associations of lifetime asthma with benzene and PM10 are particularly robust because they remain significant even among children residing at their current address since birth. Among these same children, the associations of sensitisation to pollens with VOC and PM10 reached borderline significance.

Our associations between asthma and urban pollution are consistent with results reported by recent epidemiological studies. Indeed, TAP have been associated with increased prevalence of wheezing $[8,10]$, symptoms [10], diagnosis $[8,9$, $12,13]$ and incidence $[8,15]$ of asthma, even though several studies failed to show significant associations [7, 16], most likely due to exposure misclassification. The few studies, which analysed associations of traffic exposure with allergic rhinitis 


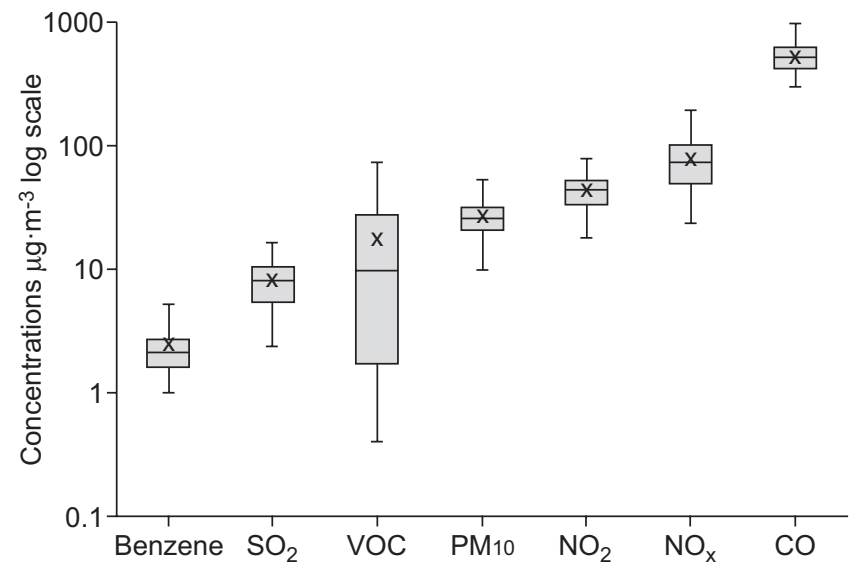

FIGURE 1. Distribution of concentrations of air pollutants. VOC: volatile organic compounds; PM10: particles with a 50\% cut-off aerodynamic diameter of $10 \mu \mathrm{m} ; \mathrm{NO}_{\mathrm{x}}$ : nitrogen oxides. The box plot represents the 25th and 75th percentiles. The horizontal line represents the median ( $x$ : mean). The whiskers extend to the minima and maxima.

$[7,10,11,14]$ and eczema $[8,14]$, however, showed mixed results. Our results for sensitisation to pollens are consistent with previous reports of associations between outdoor particles with a 50\% cut-off aerodynamic diameter of $2.5 \mu \mathrm{m}$ and sensitisation to pollens [14]. Two studies have also shown associations between allergic sensitisation and TAP, but only if the analyses were restricted to urban areas [11] or to the children additionally exposed to smoking at home [10].

Observed associations of urban pollutants with asthma and allergies are also biologically plausible [27]. Experimental studies have shown that TAP, specifically diesel exhaust particulates, one of the major contributors to PM10 in urban areas, enhance allergic response to inhalants allergens [28] and even have a direct inflammatory effect [29]. Long-term exposure to TAP mixtures, which contain small particles and organic compounds, like benzene, may provoke chronic oxidative stress and can eventually contribute to the development of asthma and allergies [30].
To our knowledge, our study is the first with model-based concentrations of such a large number of urban pollutants. Although the high correlations between the different pollutants prevents us from analysing the effect of specific pollutants [21], associations with PM10 and benzene, which is an excellent indicator of exposure to TAP mixtures, are particularly robust. Despite low outdoor levels, $\mathrm{SO}_{2}$, which is not a good proxy for traffic exposure, may still contribute to asthma, which is consistent with previous reports [31]. $\mathrm{NO}_{2}$ might be a marker of some other TAP that is responsible for increasing asthma risk [9] because indoor $\mathrm{NO}_{2}$ is not related to asthma, contrary to outdoor $\mathrm{NO}_{2}$ [11].

A major strength of our study lies in the exposure assessment. First, we used STREET 5, which is capable of modelling small-scale variations in urban pollution thus reducing misclassifications in exposure [21]. However, because pollutant concentrations were calculated only at schools exposure errors might have occurred, even if children generally live within $500 \mathrm{~m}$ of their school in urban areas in France and even if exposure at school represents an important component of their exposure experience. These exposure misclassifications would err towards an underestimation of the effects, but in no way to a bias. Secondly, we built 3-yr averaged concentrations of pollutants, whereas studies based on direct measurements generally used average concentrations obtained over several weeks to estimate long-term exposure. Moreover, to limit exposure misclassification due to moving, analyses were restricted to the children residing at their current address for $\geqslant 3$ yrs. The sensitivity analyses in longterm residents ( $\geqslant 8$ yrs at the same address) also corroborate our findings.

Another strength of our study is our use of internationally validated indicators [25] of asthma and allergic morbidities, and objective tests to determine EIA and allergic sensitisation. Moreover, because parents were not aware of the specific focus of the study on air pollution at the time the questionnaire was completed and because clinical examination was performed blindly with respect to exposure status by physicians certified in their ability to obtain comparable measures, reporting bias and misclassification are minimised.

TABLE 1 Concentrations of air pollutants calculated by STREET 5 according to the communities in the French Six Cities Study

\begin{tabular}{|c|c|c|c|c|c|c|}
\hline $\begin{array}{l}\text { Air pollutants } \\
\mu \mathrm{g} \cdot \mathrm{m}^{-3}\end{array}$ & Bordeaux ${ }^{\#}$ & Clermont-Ferrand & Créteil $^{+}$ & Marseille $^{s}$ & Reims $^{+}$ & Strasbourg ${ }^{f}$ \\
\hline VOC*** & $20.6(0.4-62.0)$ & $18.2(0.5-53.4)$ & $19.0(0.7-55.1)$ & $30.1(0.5-73.5)$ & $3.8(0.5-15.6)$ & $12.3(0.9-46.2)$ \\
\hline $\mathrm{CO} * * *$ & $521.8(303.8-873.8)$ & $560.2(424.1-872.4)$ & $619.0(504.7-870.6)$ & $637.5(433.5-988.1)$ & $381.4(304.5-509.9)$ & $584.6(407.5-893.8)$ \\
\hline $\mathrm{NO}_{2}{ }^{* \star *}$ & $37.0(17.8-59.3)$ & $44.7(29.3-63.0)$ & $56.6(51.6-78.9)$ & $50.6(40.5-62.6)$ & $30.5(22.6-40.0)$ & $42.8(34.8-58.6)$ \\
\hline $\mathrm{SO}_{2}{ }^{* * *}$ & $8.5(5.0-11.2)$ & $4.8(2.4-6.9)$ & $8.8(7.7-11.1)$ & $13.2(10.7-16.4)$ & $4.1(3.1-6.7)$ & $10.6(7.4-14.8)$ \\
\hline
\end{tabular}

Data are presented as mean concentrations among the $\mathrm{n}$ schools of the community (minimum of the concentrations of the $\mathrm{n}$ schools of the community-maximum of the concentrations of the $n$ schools of the community). VOC: volatile organic compound; $\mathrm{NO}_{x}$ : nitrogen oxides; PM10: particles with a $50 \%$ cut-off aerodynamic diameter of

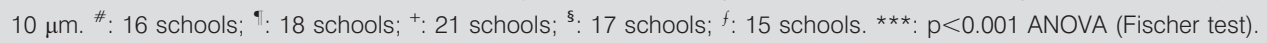




\begin{tabular}{|c|c|c|c|}
\hline Factors & Child & Mother & Father \\
\hline \multicolumn{4}{|l|}{ Socio-demographics factors } \\
\hline Age yrs & $10.4 \pm 0.7$ & $38.7 \pm 5.1$ & $41.7 \pm 6.2$ \\
\hline Male & 49.9 & & \\
\hline Weight kg & $36.1 \pm 8.1$ & & \\
\hline Height $m$ & $1.42 \pm 0.08$ & & \\
\hline $\mathrm{BMI} \mathrm{kg} \cdot \mathrm{m}^{-2}$ & $17.8 \pm 2.9$ & & \\
\hline Older siblings & 46.1 & & \\
\hline \multicolumn{4}{|l|}{ Parental education } \\
\hline Primary or secondary & & \multicolumn{2}{|c|}{51.1} \\
\hline High school and university & & \multicolumn{2}{|c|}{48.9} \\
\hline \multicolumn{4}{|l|}{ Ethnic origin } \\
\hline Metropolitan France & & 76.3 & 74.8 \\
\hline French overseas departments & & 3.2 & 2.0 \\
\hline South Europe & & 4.0 & 4.5 \\
\hline Morocco, Algeria, Tunisia & & 8.1 & 9.6 \\
\hline Sub-Saharan Africa & & 2.6 & 2.7 \\
\hline Asia & & 2.9 & 3.2 \\
\hline Other & & 3.0 & 3.2 \\
\hline Family history of allergy ${ }^{+}$ & 37.1 & & \\
\hline \multicolumn{4}{|c|}{$\begin{array}{l}\text { Potential sources of indoor pollution at } \\
\text { home }\end{array}$} \\
\hline Smoking ${ }^{\S}$ & & 43.6 & \\
\hline Mould or dampness ${ }^{f}$ & & 17.6 & \\
\hline Gas $^{\# \#}$ & & 82.1 & \\
\hline Pets $\%$ & & 47.3 & \\
\hline
\end{tabular}

Data are presented as mean \pm SD or \%. BMI: body mass index. \#: 4,907 children residing at their current address for at least three years; " : the highest educational level achieved of either parent; ${ }^{+}$: if the child's father or mother had

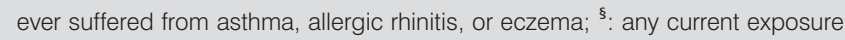
to cigarettes, pipes or cigars at home; ${ }^{f}$ : the current presence of damp or mould

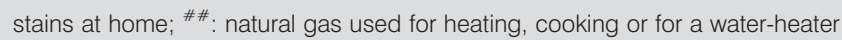
at home (except in cellar or in garage); ${ }^{\top}$ : the presence of furry or feathered pets at home (dogs, cats, rodents, ferret or polecat).

The high number of potential confounders and modifiers that were taken into account, either directly through adjustment and stratification or through the marginal model (climate, pollens, socio-cultural factors and diet), which enabled us to control the potential community effect, is another strength of our study.

In future studies we hope to be able to measure indoor pollutants even if questionnaire-derived data on indoor home air quality are available and taken into account through adjustments. Although recent studies have shown that indoor concentrations of major TAP are highly correlated with outdoor levels [32], some non-differential misclassification would probably not have been avoided.

The cross-sectional nature of our study constitutes a limitation on causal inference. However, the rank order of the 3-yr averaged concentrations calculated at schools is likely to have remained stable over the lifetimes of these children, due to the fact that no major road works were undertaken in the six

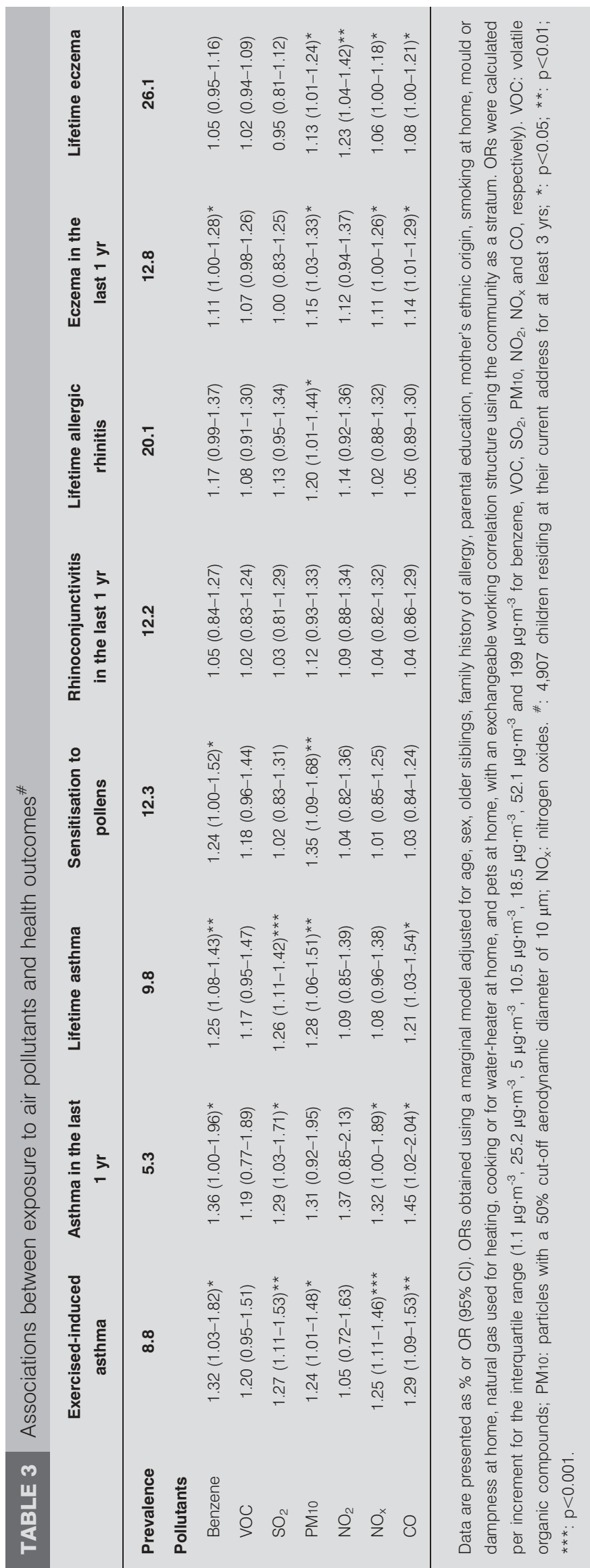



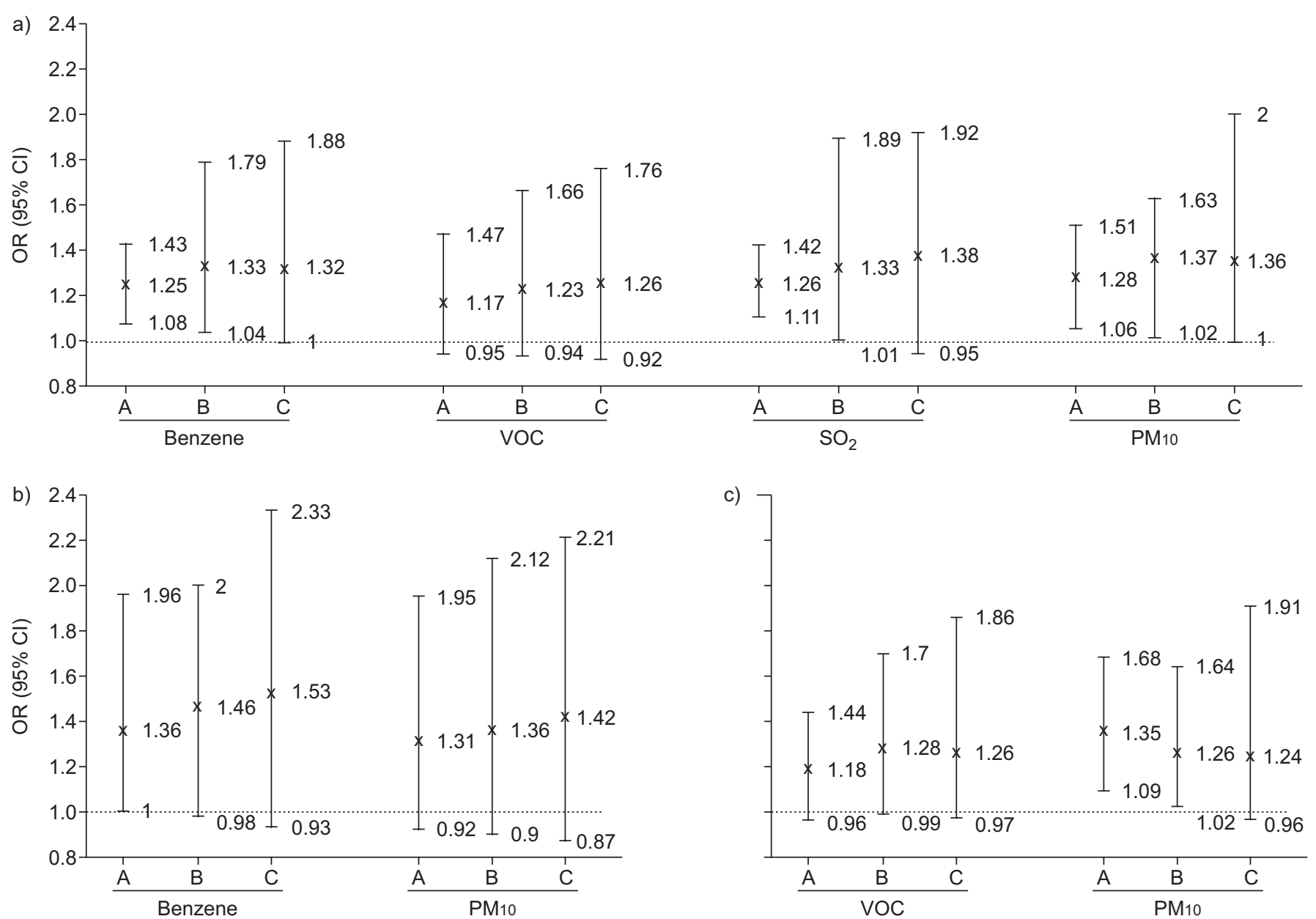

FIGURE 2. Associations between exposure to air pollutants and health outcomes according to the duration of residence at the address recorded during the survey. a) Lifetime asthma, b) asthma in the last $1 \mathrm{yr}$ and c) sensitisation to pollens. A: 4,907 children residing at their current address for $\geqslant 3$ yrs; B: 2,834 children residing at their current address for $\geqslant 8$ yrs; C: 2,213 children residing at their current address since birth. VOC: volatile organic compounds; PM10: particles with a $50 \%$ cut-off aerodynamic diameter of $10 \mu \mathrm{m}$. Data are presented as OR and $95 \% \mathrm{Cl}$ obtained with a marginal model adjusted for age, sex, older siblings, family history of allergy, parental education, mother's ethnic origin, smoking at home, mould or dampness at home, natural gas used for heating, cooking or for water-heater at home, and pets at home, with an exchangeable working correlation structure using the community as a stratum. OR were calculated per increment for the interquartile range $\left(1.1 \mu \mathrm{g} \cdot \mathrm{m}^{-3}, 25.2 \mu \mathrm{g} \cdot \mathrm{m}^{-3}, 5 \mu \mathrm{g} \cdot \mathrm{m}^{-3}, 10.5 \mu \mathrm{g} \cdot \mathrm{m}^{-3}, 18.5 \mu \mathrm{g} \cdot \mathrm{m}^{-3}, 52.1 \mu \mathrm{g} \cdot \mathrm{m}^{-3}\right.$ and $199 \mu \mathrm{g} \cdot \mathrm{m}^{-3}$ for benzene, $\mathrm{VOC}, \mathrm{SO}{ }_{2}, \mathrm{PM}_{10}, \mathrm{NO}{ }_{2}$, $\mathrm{NO}_{\mathrm{x}}$ and $\mathrm{CO}$, respectively) successively among children in group $\mathrm{A}, \mathrm{B}$ and $\mathrm{C}$. The whiskers extend to the lower bound and the upper bound of the $95 \% \mathrm{Cl}$ ( $x$ : adjusted $O R$ ).

communities between 1990 and 2000, although there may have been some changes in the absolute traffic density. Thus, the fact that the associations of benzene and PM10 with asthma, and of VOC and PM10 with sensitisation to pollens, remained robust among the children who had never moved argues in favour of an effect of these pollutants on asthma and allergies. However, distinguishing new incident asthma from a recrudescence of previous symptoms remains difficult, except in birth cohort. In a Dutch birth cohort, $\mathrm{NO}_{2}$, particles with a $50 \%$ cut-off aerodynamic diameter of $2.5 \mu \mathrm{m}$ and soot modelled through a regression model were associated with some outcomes of asthma and allergy during the first 4 yrs of life [8].

To conclude, our study showed associations between longterm exposure to urban air pollution, assessed using a dispersion model capable of capturing small-scale variations within communities, and asthma and allergies. The most robust associations were found with PM10 and benzene, primarily emitted by traffic in urban areas. These results corroborate an emerging body of evidence that traffic-related air pollution could induce asthma and allergies. Because of this emerging evidence and because of the enormous costs related to childhood asthma and allergies, the development of alternative fuels and transportation to reduce traffic emissions is called for. However, additional research is needed to study the impact of specific pollutants which are included in a complex mixture of urban emissions. Future cohort studies that assess intra-urban exposure by combining day-time activity and model-based concentrations of pollutants are needed to better understand the role of TAP on the development of asthma and allergies. 


\section{SUPPORT STATEMENT}

The French Six Cities study was supported by the National Institute for Health and Medical Research (INSERM; Programme Déterminants de la Santé), the Ministry of Health, the Environmental Programme PRIMEQUAL-PREDIT of the Ministry of Environment, the Agency for Environment and Energy Management, the French Agency for Environmental and Occupational Health Safety, the mutual insurance company of the state education system (the Mutuelle Générale de l'Education Nationale) and the French At Home Respiratory Support association (the Association Nationale pour le Traitement A Domicile de l'Insuffisance Respiratoire chronique).

\section{STATEMENT OF INTEREST}

None declared.

\section{ACKNOWLEDGEMENTS}

We are indebted to the pupils and parents, without whom the study would not have been possible. We wish to thank Education Nationale (France), the school doctors, the school principals and the teachers in the six communities. We would also like to thank G. Debotte and D. Moreau who participated in the data collection and management. We thank the specialists of Air Monitoring Networks (AIRAQ (Bordeaux), AIRPARIF (Créteil), ATMO Auvergne (Clermont-Ferrand), AIRMARAIX (Marseille), ATMO Champagne-Ardennes (Reims) and ASPA (Strasbourg, all France)) who helped us obtain data to model air pollution exposure. We thank G. Pauli (Hôpital Lyautet, Strasbourg, France), and M. Tunon de Lara and A. Taytard (University of Bordeaux 2, Bordeaux, France) who contributed to the conception and design of the study. We are also grateful to G. Mercier (French Comité National contre les Maladies Respiratoires et la Tuberculose, Paris, France), on the aegis of which the survey was conducted. Allergen extracts were kindly provided by Stallergènes Laboratoires (Antony, France).

\section{REFERENCES}

1 Asher MI, Montefort S, Bjorksten B, et al. Worldwide time trends in the prevalence of symptoms of asthma, allergic rhinoconjunctivitis, and eczema in childhood: ISAAC Phases One and Three repeat multicountry cross-sectional surveys. Lancet 2006; 368: 733-743.

2 Fenger J. Urban air quality. Atmos Environ 1999; 33: 4877-4900.

3 Heinrich J, Wichmann HE. Traffic related pollutants in Europe and their effect on allergic disease. Curr Opin Allergy Clin Immunol 2004; 4: 341-348

4 Sunyer J, Spix C, Quenel P, et al. Urban air pollution and emergency admissions for asthma in four European cities: the APHEA Project. Thorax 1997; 52: 760-765.

5 Corbo GM, Forastiere F, Dell'Orco V, et al. Effects of environment on atopic status and respiratory disorders in children. J Allergy Clin Immunol 1993; 92: 616-623.

6 Dockery DW, Speizer FE, Stram DO, et al. Effects of inhalable particles on respiratory health of children. Am Rev Respir Dis 1989, 139: 587-594.

7 Wjst M, Reitmeir P, Dold S, et al. Road traffic and adverse effects on respiratory health in children. BMJ 1993; 307: 596-600.

8 Brauer M, Hoek G, Smit HA, et al. Air pollution and development of asthma, allergy and infections in a birth cohort. Eur Respir J 2007; 29: 879-888.

9 Gauderman WJ, Avol E, Lurmann F, et al. Childhood asthma and exposure to traffic and nitrogen dioxide. Epidemiology 2005; 16 737-743.

10 Nicolai T, Carr D, Weiland SK, et al. Urban traffic and pollutant exposure related to respiratory outcomes and atopy in a large sample of children. Eur Respir J 2003; 21: 956-963.
11 Kramer U, Koch T, Ranft U, et al. Traffic-related air pollution is associated with atopy in children living in urban areas. Epidemiology 2000; 11: 64-70.

12 Kim JJ, Smorodinsky S, Lipsett M, et al. Traffic-related air pollution near busy roads: the East Bay Children's Respiratory Health Study. Am J Respir Crit Care Med 2004; 170: 520-526.

13 Zmirou D, Gauvin S, Pin I, et al. Traffic related air pollution and incidence of childhood asthma: results of the Vesta case-control study. J Epidemiol Community Health 2004; 58: 18-23.

14 Janssen NA, Brunekreef B, van Vliet $\mathrm{P}$, et al. The relationship between air pollution from heavy traffic and allergic sensitization, bronchial hyperresponsiveness, and respiratory symptoms in Dutch schoolchildren. Environ Health Perspect 2003; 111: 1512-1518.

15 Jerrett $M$, Shankardass $\mathrm{K}$, Berhane $\mathrm{K}$, et al. Traffic-related air pollution and asthma onset in children: a prospective cohort study with individual exposure measurement. Environ Health Perspect 2008; 116: 1433-1438.

16 Wilkinson P, Elliott P, Grundy C, et al. Case-control study of hospital admission with asthma in children aged 5-14 years: relation with road traffic in north west London. Thorax 1999; 54: 1070-1074.

17 Jerrett M, Arain A, Kanaroglou P, et al. A review and evaluation of intraurban air pollution exposure models. J Expo Anal Environ Epidemiol 2005; 15: 185-204.

18 Brauer M, Hoek G, van Vliet $P$, et al. Estimating long-term average particulate air pollution concentrations: application of traffic indicators and geographic information systems. Epidemiology 2003; 14: 228-239.

19 Carr D, von Ehrenstein O, Weiland S, et al. Modeling annual benzene, toluene, $\mathrm{NO}_{2}$, and soot concentrations on the basis of road traffic characteristics. Environ Res 2002; 90: 111-118.

20 Reungoat $\mathrm{P}$, Chiron M, Gauvin S, et al. Retrospective assessment of exposure to traffic air pollution using the ExTra index in the VESTA French epidemiological study. I Expo Anal Environ Epidemiol 2005; 15: 524-533.

21 Pénard-Morand C, Schillinger C, Armengaud A, et al. Assessment of schoolchildren's exposure to traffic-related air pollution in the French Six Cities Study using a dispersion model. Atmos Environ 2006; 40: 2274-2287.

22 Morgenstern V, Zutavern A, Cyrys J, et al. Respiratory health and individual estimated exposure to traffic-related air pollutants in a cohort of young children. Occup Environ Med 2007; 64: $8-16$.

23 Pénard-Morand C, Charpin D, Raherison C, et al. Long-term exposure to background air pollution related to respiratory and allergic health in schoolchildren. Clin Exp Allergy 2005; 35: 1279-1287.

24 Annesi-Maesano I, Moreau D, Caillaud D, et al. Residential proximity fine particles related to allergic sensitisation and asthma in primary school children. Respir Med 2007; 101 1721-1729.

25 Weiland SK, Bjorksten B, Brunekreef B, et al. Phase II of the International Study of Asthma and Allergies in Childhood (ISAAC II): rationale and methods. Eur Respir J 2004; 24: 406-412.

26 Worldwide variation in prevalence of symptoms of asthma, allergic rhinoconjunctivitis, and atopic eczema: ISAAC. The International Study of Asthma and Allergies in Childhood (ISAAC) Steering Committee. Lancet 1998; 351: 1225-1232.

27 Penard-Morand C, Annesi-Maesano I. [Allergic respiratory diseases and outdoor air pollution]. Rev Mal Respir 2008; 25 1013-1026.

28 Diaz-Sanchez D, Penichet-Garcia M, Saxon A. Diesel exhaust particles directly induce activated mast cells to degranulate and increase histamine levels and symptom severity. J Allergy Clin Immunol 2000; 106: 1140-1146. 
29 Salvi S, Blomberg A, Rudell B, et al. Acute inflammatory responses in the airways and peripheral blood after short-term exposure to diesel exhaust in healthy human volunteers. Am J Respir Crit Care Med 1999; 159: 702-709.

$30 \mathrm{Nel}$ A. Atmosphere. Air pollution-related illness: effects of particles. Science 2005; 308: 804-806.
31 Chen TM, Gokhale J, Shofer S, et al. Outdoor air pollution: nitrogen dioxide, sulfur dioxide, and carbon monoxide health effects. Am J Med Sci 2007; 333: 249-256.

32 Fischer PH, Hoek G, Van Reeuwijk H, et al. Traffic-related differences in outdoor and indoor concentrations of particles and volatile organic compounds in Amsterdam. Atmos Environ 2000; 34: 3713-3722. 